Acta Mechanica 189, 241-243 (2007)

DOI 10.1007/s00707-006-0422-8

Printed in The Netherlands

Acta Mechanica

\title{
Erratum
}

\section{Obliquely incident surface waves in shallow water of slowly varying depth}

(DOI 10.1007/s00707-006-0392-x)

\author{
C. A. van Duin, De Bilt, The Netherlands
}

Published online: January 25, 2007 C Springer-Verlag 2007

The analysis below Fig. 1 in Sect. 3 of the original paper is not correct. Here, a revised version is presented. This should replace the original analysis at the end of Sect. 3.

The composite shelf, induced by the primary wave, cannot propagate further than the characteristics $\theta_{+}=0, \theta_{-}=0$. In other words, this shelf is confined to a wedge-shaped region in the $(\zeta, \xi)$-plane, bounded above by the characteristics (Fig. 1). Accordingly, Eq. (44) is integrated along $C$ between the initial point $A$ and the end point $B$. This leads to the equation

$$
\begin{aligned}
& \int_{\hat{\zeta}(\tau)}^{\bar{\zeta}(\tau)} \hat{\eta}_{\tau}\left(\zeta, \xi_{0}, \tau\right) \mathrm{d} \zeta+\int_{\hat{\zeta}(\tau)}^{\bar{\zeta}(\tau)} h \hat{\phi}_{\xi \xi}\left(\zeta, \xi_{0}, \tau\right) \mathrm{d} \zeta+\left[h \hat{\phi}_{\zeta}\right]_{+}-\left[\hat{\phi}_{\zeta}\right]_{-} \\
& \quad=\frac{\mathrm{d}}{\mathrm{d} \tau} \int_{\hat{\zeta}(\tau)}^{\bar{\zeta}(\tau)} \hat{\eta}\left(\zeta, \xi_{0}, \tau\right) \mathrm{d} \zeta+\int_{\tilde{\zeta}(\tau)}^{\bar{\zeta}(\tau)} h \hat{\phi}_{\xi \xi}\left(\zeta, \xi_{0}, \tau\right) \mathrm{d} \zeta+\left[h \hat{\phi}_{\zeta}-k^{-1} \hat{\eta}\right]_{+}-\left[\hat{\phi}_{\zeta}+k_{0}^{-1} \hat{\eta}\right]_{-}=0,
\end{aligned}
$$

where $[. .]_{+}$and $[. .]_{-}$denote evaluation along the characteristics $\theta_{+}=0$ and $\theta_{-}=0$, respectively.

Since the contribution of the right-going shelf (41) to mass transfer is negligibly small, the mass loss of the primary wave is completely transferred to the composite shelf. Based on a comparison of (32), (40) and (47) it is then found that the requirement that mass is conserved necessarily implies that

$\left[h \hat{\phi}_{\zeta}-k^{-1} \hat{\eta}\right]_{+}-\left[\hat{\phi}_{\zeta}+k_{0}^{-1} \hat{\eta}\right]_{-}=m_{0} k_{0}^{3 / 2} h_{+} k_{+}^{2} \frac{\mathrm{d}}{\mathrm{d} \tau}\left(h_{+}^{-1 / 2} k_{+}^{-3 / 2}\right)$.

When $h$ and $k$ are expressed in terms of the variables $\theta_{+}$and $\theta_{-}$, it is found that these are of the form $h=\hat{h}\left(\theta_{-}-\theta_{+}\right), k=\hat{k}\left(\theta_{-}-\theta_{+}\right)$, which implies that $h_{+}=\hat{h}\left(\theta_{-}\right), k_{+}=\hat{k}\left(\theta_{-}\right)$. Clearly, the right-hand side of Eq. (48), with $\mathrm{d} / \mathrm{d} \tau=-2 \mathrm{~d} / \mathrm{d} \theta_{-}$, is independent of $\theta_{+}$. The first term on the left-hand side of Eq. (48), with $\hat{\phi}$ and $\hat{\eta}$ expressed in terms of $\theta_{+}$and $\theta_{-}$, is also independent of $\theta_{+}$. This leads to the conclusion that the second term is of the form

$\left[\hat{\phi}_{\zeta}+k_{0}^{-1} \hat{\eta}\right]_{-}=k_{0}^{-1}\left[\left(1+k_{0}^{2}\right) \hat{\phi}_{\theta_{+}}+\ell^{2} \hat{\phi}_{\theta_{-}}\right]_{-}=m_{0} F_{0}$,

where the constant $F_{0}$ is still to be determined. 
From (48) and (49) we obtain

$\left[h \ell^{2} \hat{\phi}_{\theta_{+}}+\left(1+h k^{2}\right) \hat{\phi}_{\theta_{-}}\right]_{+}=-m_{0} k_{0}^{3 / 2} h_{+} k_{+}^{3} \frac{\mathrm{d}}{\mathrm{d} \tau}\left(h_{+}^{-1 / 2} k_{+}^{-3 / 2}\right)-m_{0} k_{+} F_{0}$.

It is assumed that $\mathrm{d} h / \mathrm{d} \zeta$ is continuous. Then $\mathrm{d} h / \mathrm{d} \zeta=0$ at $\zeta=0$, and the first term on the right-hand side of Eq. (50) vanishes as $\tau \downarrow 0$ if the path of integration is chosen along the $\zeta$-axis. Since this term is evaluated at $\theta_{+}=0$, and $\bar{\zeta}(\tau) \downarrow 0$ as $\tau \downarrow 0$, this corresponds to the limit $\theta_{-} \uparrow 0$. From (49) and (50) it then follows that

$\hat{\phi}_{\theta_{-}}=-m_{0} F_{0} / 2 k_{0}, \quad \hat{\phi}_{\theta_{+}}=m_{0} F_{0} / 2 k_{0} \quad$ at $\quad \theta_{+}=0, \quad \theta_{-}=0$,

which implies that $\hat{\eta}=0$ at $\theta_{+}=0, \theta_{-}=0$. In addition, the right-going wave component of the composite shelf should vanish in the region $\zeta<0$, i.e., $\hat{\eta}_{\theta_{+}}=0$ in the region of uniform depth. Combining these results, we obtain

$[\hat{\eta}]_{-}=0$.

The equation for the potential, derived from (43)-(45), is of the form

$\hat{\phi}_{\theta_{+} \theta_{-}}=(4 h k)^{-1}\left[(h k)_{\theta_{+}}-(h k)_{\theta_{-}}\right]\left(\hat{\phi}_{\theta_{+}}-\hat{\phi}_{\theta_{-}}\right)$.

Along the characteristic $\theta_{+}=0$, Eq. (53) converts into an ordinary differential equation. Using (50) to eliminate the term $\left[\hat{\phi}_{\theta_{-}}\right]_{+}$, the resulting equation reads

$$
\begin{gathered}
\frac{\mathrm{d}}{\mathrm{d} \theta_{-}}\left[\hat{\phi}_{\theta_{+}}\right]_{+}+\left(2 h_{+} k_{+}\left(1+h_{+} k_{+}^{2}\right)\right)^{-1} \frac{\mathrm{d}}{\mathrm{d} \theta_{-}}\left(h_{+} k_{+}\right)\left[2\left[\hat{\phi}_{\theta_{+}}\right]_{+}\right. \\
\left.+m_{0} k_{+} F_{0}-2 m_{0} k_{0}^{3 / 2} h_{+} k_{+}^{3} \frac{\mathrm{d}}{\mathrm{d} \theta_{-}}\left(h_{+}^{-1 / 2} k_{+}^{-3 / 2}\right)\right]=0 .
\end{gathered}
$$

Writing Eq. (54) in the form $\mathrm{d}\left[\hat{\phi}_{\theta_{+}}\right]_{+} / \mathrm{d} \theta_{-}+\alpha\left(\theta_{-}\right)\left[\hat{\phi}_{\theta_{+}}\right]_{+}+\beta\left(\theta_{-}\right)=0$, the initial condition (51) leads to the solution

$\left[\hat{\phi}_{\theta_{+}}\right]_{+}=\left[m_{0} F_{0} / 2 k_{0}-\int_{0}^{\theta_{-}} \beta(s) \exp \left(\int_{0}^{s} \alpha(u) \mathrm{d} u\right) \mathrm{d} s\right] \exp \left(-\int_{0}^{\theta_{-}} \alpha(s) \mathrm{d} s\right)$.

The expression for $\left[\hat{\phi}_{\theta_{-}}\right]_{+}$is determined from (50) and (55). This leads to the result

$$
\begin{aligned}
{[\hat{\eta}]_{+}=} & \left(1+h_{+} k_{+}^{2}\right)^{-1}\left[2 m_{0} k_{0}^{3 / 2} h_{+} k_{+}^{3} \frac{\mathrm{d}}{\mathrm{d} \theta_{-}}\left(h_{+}^{-1 / 2} k_{+}^{-3 / 2}\right)-m_{0} k_{+} F_{0}\right] \\
& +\frac{2 h_{+} k_{+}^{2}}{\left(1+h_{+} k_{+}^{2}\right)}\left[m_{0} F_{0} / 2 k_{0}-\int_{0}^{\theta_{-}} \beta(s) \exp \left(\int_{0}^{s} \alpha(u) \mathrm{d} u\right) \mathrm{d} s\right] \exp \left(-\int_{0}^{\theta_{-}} \alpha(s) \mathrm{d} s\right) .
\end{aligned}
$$

The mass carried by the composite shelf along $C$, expressed by the integral

$$
\int_{\hat{\zeta}(\tau)}^{\bar{\zeta}(\tau)} \hat{\eta}\left(\zeta, \xi_{0}, \tau\right) \mathrm{d} \zeta
$$

must be bounded as $\tau \rightarrow \infty$. This necessarily implies that $[\hat{\eta}]_{+} \rightarrow 0$ in this limit, which corresponds to the requirement that $[\hat{\eta}]_{+} \rightarrow 0$ as $\theta_{-} \rightarrow-\infty$. Then the constant $F_{0}$ in (56) is uniquely determined.

The requirement that mass is conserved leads to the expressions (52) and (56) for $[\hat{\eta}]_{-}$and $[\hat{\eta}]_{+}$, which prescribe the solution of Eq. (46) along the characteristics $\theta_{-}=0$ and $\theta_{+}=0$, 
respectively. The problem thus posed has a unique solution [16]. Finally, it is noted that the physically relevant region is confined to the third quadrant in the $\left(\theta_{-}, \theta_{+}\right)$-plane.

Author's address: C. A. van Duin, Department of Oceanography, Royal Netherlands Meteorological Institute, 3730 AE De Bilt, The Netherlands (E-mail: ca.ew.vanduin@hetnet.nl) 